\title{
Aplicabilidade de Sistemas de Informações Geográficas (SIGs) livres nas ciências ambientais: o uso do QGIS
}

\section{Leandro Obadowiski Bruno}

Secretaria de Estado do Meio Ambiente. Palácio Paiaguás, Rua C. Cuiabá-MT. Brasil. (CEP 78049-913). E-mail: obadowiski@gmail.com.

Resumo. Os Sistemas de Informações Geográficas (SIGs) tornaramse componentes essenciais em variados campos de pesquisas e tarefas científicas dentro do domínio das ciências ambientais. Nesta categoria de aplicativos, destacam-se os chamados softwares livres por demostrarem potencialidades promissoras. Este trabalho abordou a utilização das geotecnologias livres com enfoque no uso do QGIS. Utilizou-se como ferramenta a revisão de literatura a partir de estudos relacionados às ciências ambientais. Conclui-se que o software livre pode ser considerado uma abrangente plataforma de processamento e divulgação do conhecimento.

Palavras-chave: Ambiente; Recursos hídricos; Geoprocessamento.

Abstract. Applicability of free Geographical Information Systems (GIS) in environmental sciences. Geographic information systems (GIS) have become essential components in a variety of research fields and scientific tasks within the field of environmental sciences. In this category of applications, stand out the so-called free software for demonstrating promising potentialities. This work addresses a use of free geotechnologies with a focus without QGIS. It was used as the literature review from studies related to the environmental sciences. It is concluded that free software can be considered a comprehensive platform for processing and disseminating knowledge.

Keywords: Environment; Water resources; Geoprocessing.

Recebido:

05/10/2017

Aceito:

$18 / 11 / 2017$

Publicado:

31/12/2017

Acesso aberto

Artigo completo

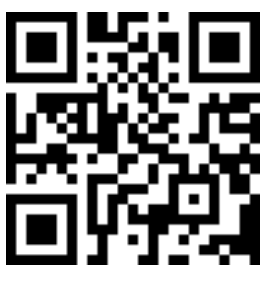

ORCID

(ㄱ) 0000-0002-8306-5533

Leandro Obadowiski Bruno

\section{Introdução}

Nas últimas décadas inúmeros projetos voltados ao desenvolvimento de Sistemas de Informações Geográficas (SIGs) iniciaram-se devido ao avanço tecnológico e computacional. Atualmente, os chamados softwares livres vem se destacando nesta categoria de aplicativos por demonstrarem potencialidades promissoras em variados campos de pesquisas e tarefas científicas dentro do domínio das ciências ambientais (Fan et al., 2015; Landuyt et al., 2015; Swain et al., 2015). $\begin{array}{cc}\text { Como exemplo, é possível citar } \\ \text { alguns } & \text { softwares }\end{array}$ independentes ou integrados, como OSSIM, Orfeo ToolBox, Opticks e GRASS GIS (Rocchini et al., 2017). Dentre tantas alternativas livres disponíveis, destaca-se o software QGIS (QGIS Development Team, 2017) em razão da sua crescente utilização em todo o mundo e vasto material de apoio aos utilizadores.

O Qgis é um software livre para Sistema de Informação Geográfica, incubado pela Open Source Geospatial Foundation (OSGeo) e impulsionado por um grupo ativo de desenvolvedores voluntários que regularmente lançam 
atualizações e correções para os problemas verificados neste aplicativo. É utilizado em ambientes acadêmicos e profissionais, caracterizando-se por oferecer um número crescente de recursos nativos e "plugins" que podem ser desenvolvidos por qualquer usuário que saiba programar em $\mathrm{C}++$ ou Python. Suporta uma ampla gama de formatos geoespaciais, tais como vetores, rasters e bases de dados.

O software livre apresenta-se como uma afirmação de liberdade, no sentido de independência de expressão (FSF, 2015). Permitem-se adaptações ou modificações em seu código de forma espontânea, sem que haja a necessidade de solicitar permissão ao seu proprietário (Milaré et al., 2016).

Há uma demanda crescente na comunidade científica, assim como dos órgãos públicos, em reproduzir o conjunto exato dos dados e dos códigos utilizados em estudos voltados às ciências ambientais. Além disso, muitos grupos de pesquisas e empresas privadas, particularmente aquelas de pequeno porte, não dispõem de recursos financeiros suficientes para adquirir e atualizar as licenças requeridas para a utilização dos softwares proprietários pagos. Desse modo, a aplicação do software livre tem aumentado consideravelmente.

Objetivou-se neste trabalho realizar um levantamento na literatura acerca da utilização dos sistemas de informações geográficas livres, com enfoque no QGIS, para estudos relacionados às ciências ambientais.

\section{Material e métodos}

Neste estudo foi realizada uma revisão da literatura sobre a utilização de geotecnologias livres no campo das ciências ambientais. Para isso, efetuaram-se buscas de artigos publicados no banco de dados Science Direct, Wiley Online Library e Springer Link. Priorizaram-se trabalhos datados dos últimos cinco anos (2012-2017) envolvendo o uso do QGIS. Através do procedimento metodológico foram selecionados 22 artigos pertinentes ao tema.

\section{Resultados e discussão}

\section{Afinal, o que é o QGIS?}

O QGIS é um SIG livre licenciado pela Licença Pública Geral - GNU (QGIS Development Team, 2017) desenvolvido em 2002 como alternativa à interface do GRASS GIS (Ellul, 2012). Trata-se de um projeto oficial da Open Source Geospatial Foundation - OSGeo (OSGeo, 2017) e impulsionado por voluntários. Caracterizase por executar funções avançadas quando integrado a outros pacotes livres como o PostGIS, GRASS, SAGA, MapServer e outros (Figura 1). Apresenta ainda boas possibilidades de personalização, como idiomas e plugins. O QGIS funciona em Linux, Unix, Mac OSX, Windows e Android e suporta inúmeros formatos de vetores, rasters, bases de dados e funcionalidades.

\section{Por que falar sobre o QGIS?}

Estudos como o de Steiniger e Hay (2009) apontam que o QGIS é considerado uma das mais promissoras plataformas livres para SIG, tendo em vista a interface eficaz e intuitiva.

Chen et al. (2010), ao comparar trinta e uma soluções livres para SIG, verificaram que o QGIS superou os demais softwares analisados e suas funcionalidades foram consideradas adequadas para a maioria das aplicações no gerenciamento dos recursos hídricos. O QGIS também pode ser considerado uma das melhores opções no contexto de gerenciamento de desastres, em razão da sua integração à diversos pacotes de softwares livres (Leiding e Teeuw, 2015).

Lobo et. al (2012) enfatizam que o QGIS se tornou uma plataforma de exibição de informações geográficas com amplos formatos geoespaciais de leitura e escrita, associado a interface minimalista e eficiente e com capacidade de exibição de camadas de servidores remotos. Além do mais, ao contrário de conjuntos de software proprietários, o QGIS está disponível gratuitamente para uso e não requerer taxas para a ativação e utilização de suas extensões (Yu et. al 2016). 


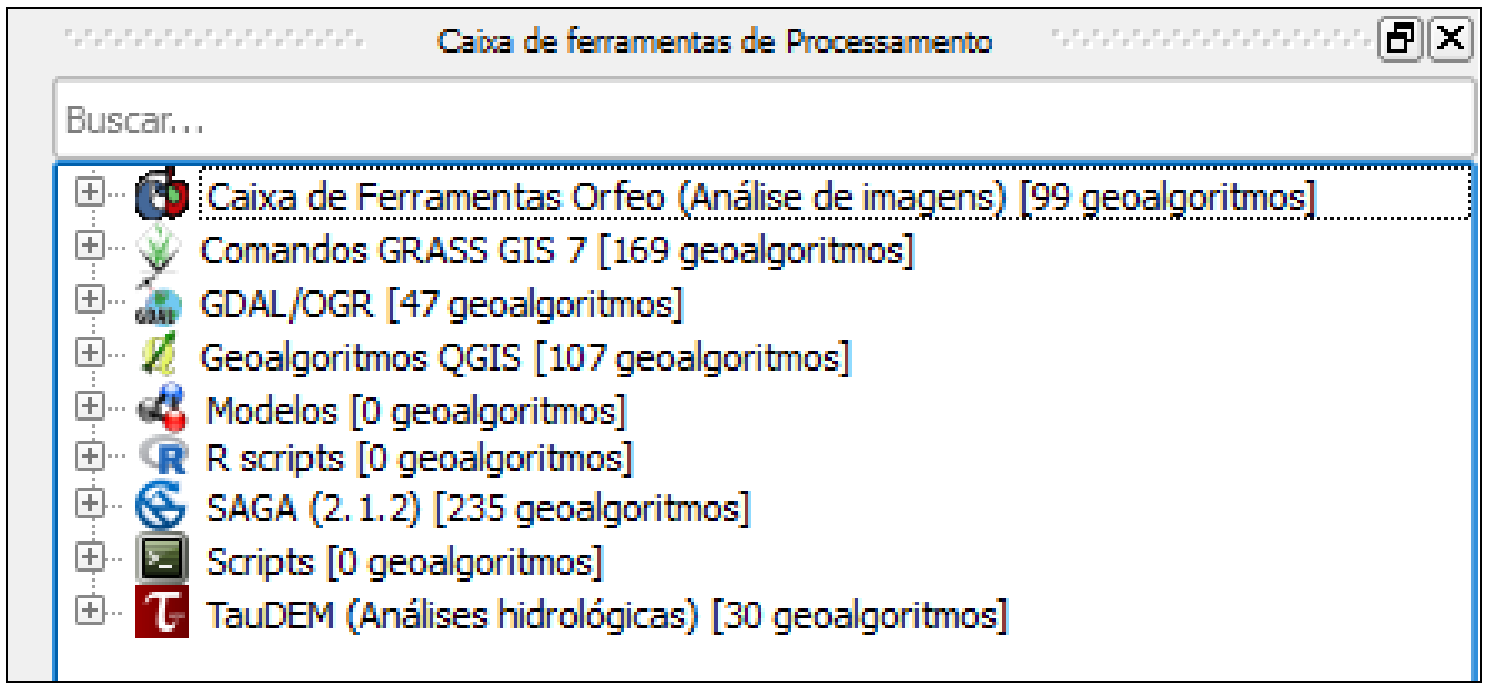

Figura 1. Pacotes de softwares livres integrados ao QGIS.

Para que se destinam os plugins?

Os plugins, também denominados

de complementos são ferramentas específicas agregadas ao QGIS, por meio dos quais são efetuadas análises específicas (Figura 2).

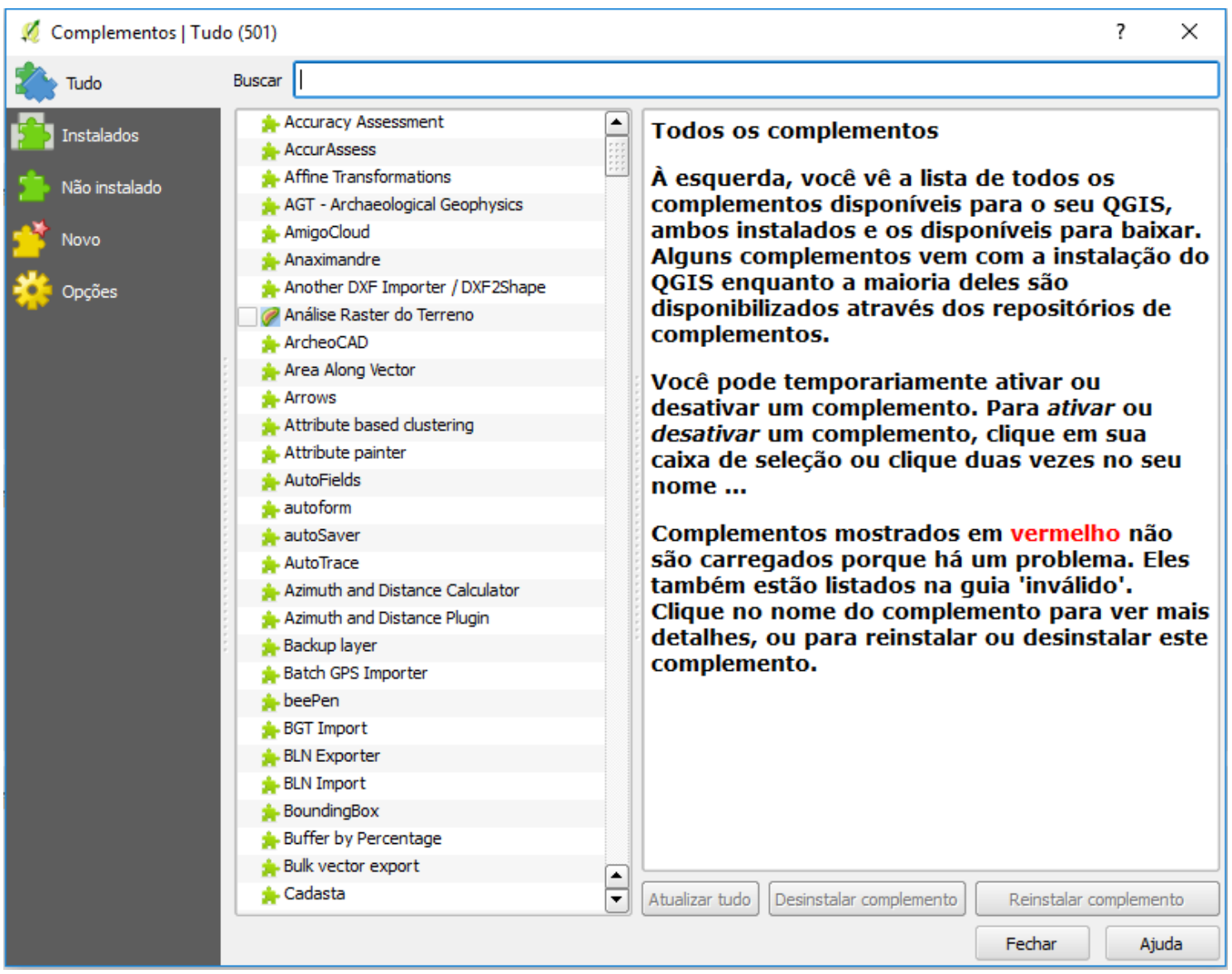

Figura 2. Índice de complementos do QGIS. 
Os complementos são escritos em linguagem Python ou $\mathrm{C}++$ potencializando as capacidades e funcionalidades do software. Neste quesito é citado que:

O QGIS possui um componente específico dedicado ao desenvolvimento de plugins. Do ponto de vista dos desenvolvedores, este aspecto tem duas vantagens principais: (a) evita trabalhar diretamente no aplicativo central, o que geralmente pode levar a problemas relacionados à estabilidade geral do software e à complexidade do código; (b) simplifica o trabalho dos desenvolvedores e permite que eles se concentrem em suas necessidades específicas. Para os usuários, o mecanismo de plugin possui duas vantagens principais: (c) 0 download é restrito às capacidades requeridas, que libera todo o aplicativo tornando-o menos pesado em termos de recursos informáticos; (d) a interface do usuário é simplificada, uma vez que apenas os ícones básicos do núcleo e os ícones do plugin carregado são exibidos na interface gráfica do usuário ao mesmo tempo (Poizot e Méar, 2010).

\section{Exemplos de utilização do QGIS nas ciências ambientais \\ Uma série de estudos tem} apresentado potencialidades do QGIS. Por exemplo, Shane et al. (2016) avaliaram o potencial de bioenergia proveniente dos resíduos sólidos gerados na Zâmbia. A pesquisa contribui significativamente para a formulação de uma política de bioenergia integrada, sustentável e educativa naquele país. Polo et al. (2015) utilizaram o QGIS para identificar as áreas potencialmente viáveis para à implantação de usinas de energia solar no Vietnã. Lee et al. (2014) produziram um conjunto de dados de alta resolução de precipitação para subsidiar estudos hidrológicos e climatológicos em bacias hidrográficas com baixa disponibilidade de dados pluviométricos. $\mathrm{O}$ QGIS também é amplamente utilizado na gestão de catástrofes, desastres naturais e respostas humanitárias (Balkaya et al., 2015; Khalil et al., 2015; Leidig e Teeuw, 2015).

\section{Estudos de caso}

Dile et al. (2016) desenvolveram uma interface da ferramenta de modelagem de bacias hidrográficas, a The Soil and Water Assessment Tool (SWAT), para ser acoplada ao QGIS, denomina QSWAT. Nesta plataforma foram aprimorados alguns recursos como a fusão de sub-bacias e a visualização estática e dinâmica da saída dos dados. O estudo de caso foi realizado na Bacia Gumera (Etiópia), para demonstração da ferramenta, a qual apresentou resultado bem-sucedido. $\mathrm{O}$ QSWAT pode ser considerado uma ferramenta de extrema importância para a comunidade científica com disponibilidades e funcionalidades melhoradas em comparação a outras opções de interfaces do SWAT.

Através da aplicação de sensoriamento remoto e SIG Nino et al. (2017) avaliaram os potenciais da vida selvagem e outros atrativos da floresta Munessa-Shashemene e seu entorno, na Etiópia. Conforme resultados obtidos, a área de estudos se mostrou muito importante para os recursos hídricos, conservação da vida selvagem e para o desenvolvimento do ecoturismo.

Tebano et al. (2017) desenvolveram um pacote de ferramentas para análises geomorfológicas de bacias hidrográficas, denominado QMorphoStream. Exemplos de aplicações foram apresentados em duas bacias do sul da Itália com o propósito de desenvolver métodos para estimativa do aporte de sedimentos naquele país.

Lakota e Stajnko (2013) associaram informações geoespaciais à dados de resíduos sólidos na Eslovênia. Exemplos práticos mostraram as informações coletados para os municípios de Pomurje, colaborando para a o gerenciamento dos biogênicos e contribuindo para o uso de fontes alternativas de energia. 


\section{Conclusões}

Foram avaliados diversos artigos científicos internacionais, priorizando-se os trabalhos com até cinco anos de publicação, o que conferiu inovação e tecnologia ao estudo. Relatou-se, desse modo, a utilização das geotecnologias livres em variados ramos das ciências ambientais.

O QGIS se mostrou um software robusto e versátil, caracterizado pela acessibilidade ao código fonte. Este fator facilita a construção de ferramentas específicas, ao mesmo tempo em que simplifica a utilização do software.

As liberdades de uso, uma ampla gama de pacotes livres integrados e a disponibilização sem custos certamente contribuem para a aceitação do QGIS no meio acadêmico e coorporativo.

Espera-se que este trabalho seja de grande valia na difusão do conhecimento e na amplificação de alternativas para o uso de SIG em projetos desenvolvidos por estudantes, professores e demais profissionais no campo das ciências ambientais.

\section{Declaração de conflito de interesses}

Os autores declaram não haver conflito de interesses.

\section{Referências}

Balkaya, C.; Casciati, F.; Casciati, S.; Faravelli, L.; Vece, M. Real-time identification of disaster areas by an open-access vision-based tool. Advances in Engineering Software, v. 88, p. 83-90, 2015. https://doi.org/10.1016/j.advengsoft.2015.06.002

Chen, D.; Shams, S.; Carmona-Moreno, C.; Leone, A. Assessment of open source GIS software for water resources management in developing countries. Journal of HydroEnvironment Research, v. 4, n. 3, p. 253-264, 2010. https://doi.org/10.1016/j.jher.2010.04.017

Dile, Y. T.; Daggupati, P.; George, C.; Srinivasan, R.; Arnold, J. Introducing a new open source GIS user interface for the SWAT model. Environmental Modelling \& Software, v. 85, p. 129-138, 2016. https://doi.org/10.1016/j.envsoft.2016.08.004
Ellul, Claire. Can Free (and Open Source) Software and Data be Used to Underpin a Self-Paced Tutorial on Spatial Databases? Transactions in GIS, v. 16, n. 4, p. 435-454, $2012 . \quad$ https://doi.org/10.1111/j.14679671.2012.01321.x

Fan, F. M.; Fleischmann, A. S.; Collischonn, W.; Ames, D. P.; Rigo, D. Large-scale analytical water quality model coupled with GIS for simulation of point sourced pollutant discharges. Environmental Modelling \& Software, v. 64, p. 58-71, 2015. https://doi.org/10.1016/j.envsoft.2014.11.012

FSF - Free Software Fundation. O que é software livre? 2015. Disponível em: $<$ http://www.gnu.org/philosophy/free-sw.html>. Acesso em: 11 Out. 2015.

Khalil, A. E.; Deif, A.; Abdel Hafiez, H. E. Seismic hazard assessments at Islamic Cairo, Egypt. Journal of African Earth Sciences, $\begin{array}{lll}\text { v. 112, } & \text { Part A, } & \text { p. 287-298, }\end{array}$ https://doi.org/10.1016/j.jafrearsci.2015.09.008

Lakota, M.; Stajnko, D. Using of GIS Tools for Analysis of Organic Waste Management in Slovenia Region Pomurje. Procedia Technology, v. 8, p. 570-574, 2013. https://doi.org/10.1016/j.protcy.2013.11.081

Landuyt, D.; Van Der Biest, K.; Broekx, S.; Staes, J.; Meire, P.; Goethals, P. L. M. A GIS plug-in for Bayesian belief networks: towards a transparent software framework to assess and visualise uncertainties in ecosystem service mapping. Environmental Modelling \& $\begin{array}{llll}\text { Software, } & \text { v. } 71, \quad \text { p. 30-38, } 2015 .\end{array}$ https://doi.org/10.1016/j.envsoft.2015.05.002

Lee, W.-S.; Chun, J. A.; Kang, K. Development and application of GIS-based PRISM integration through a plugin approach. Journal of Hydrology, v. 513, p. 58-67, 2014. https://doi.org/10.1016/j.jhydrol.2014.03.029

Leidig, M.; Teeuw, R. Free software: a review, in the context of disaster management. International Journal of Applied Earth Observation and Geoinformation, v. 42, p. 49-56, 2015. https://doi.org/10.1016/j.jag.2015.05.012

Lobo, A.; Ara, F.; Baró, F.; Camino, C. Geospatial analysis for conservation: applications with open-source software in the Natural Parks of Barcelona. Applied Geomatics, v. 4, n. 2, p. 113-122, 2012. https://doi.org/10.1007/s12518-012-0079-z

Milaré, G.; Da Silva, N. M.; Paranhos Filho, A. C. Cenário do uso de software livre em Sistemas de Informações Geográficas (SIG) no Brasil. 
Anuário do Instituto de Geociências - UFRJ, v. 39, n. 3, $\quad$ p. 111-115, 2016. http://dx.doi.org/10.11137/2016_3_111_115

Narra, P.; Coelho, C.; Sancho, F.; Palalane, J. CERA: an open-source tool for coastal erosion risk assessment. Ocean \& Coastal Management, v. 142, p. 1-14, 2017. https://doi.org/10.1016/j.ocecoaman.2017.03.013

Nino, K.; Mamo, Y.; Mengesha, G.; Kibret, K. S. GIS based ecotourism potential assessment in Munessa Shashemene Concession Forest and its surrounding area, Ethiopia. Applied Geography, v. 82, p. 48-58, 2017. https://doi.org/10.1016/j.apgeog.2017.02.010

OSGeo. Overview. 2017. Disponível em: $<$ http://www.osgeo.org/>. Acesso em: $16 \mathrm{fev}$. 2017.

Poizot, E.; Méar, Y. Using a GIS to enhance grain size trend analysis. Environmental

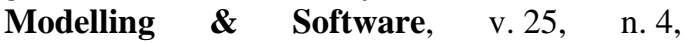
p. 513-525, 2010. https://doi.org/10.1016/j.envsoft.2009.10.002

Polo, J.; Bernardos, A.; Navarro, A. A.; Fernandez-Peruchena, C. M.; Ramírez, L.; Guisado, M. V.; Martínez, S. Solar resources and power potential mapping in Vietnam using satellite-derived and GIS-based information. Energy Conversion and Management, v. 98, p. 348-358, 2015. https://doi.org/10.1016/j.enconman.2015.04.016 QGIS Development Team. QGIS Geographic Information System. Open Source Geospatial Foundation. 2017. Disponível em: $<$ http://qgis.osgeo.org/pt_BR/site/>. Acesso em: 15 Fev. 2017.

Rocchini, D.; Petras, V.; Petrasova, A.; Chemin, Y.; Ricotta, C.; Frigeri, A.; Landa, M.;
Marcantonio, M.; Bastin, L.; Metz, M.; Delucchi, L.; Neteler, M. Spatio-ecological complexity measures in GRASS GIS. Computers \& Geosciences, v. 104, p. 166-176, 2017. https://doi.org/10.1016/j.cageo.2016.05.006

Shane, A.; Gheewala, S. H.; Fungtammasan, B.; Silalertruksa, T.; Bonnet, S.; Phiri, S. Bioenergy resource assessment for Zambia. Renewable and Sustainable Energy Reviews, v. 53, p. 93-104, 2016. https://doi.org/10.1016/j.rser.2015.08.045

Steiniger, S.; Hay, G. J. Free and open source geographic information tools for landscape ecology. Ecological Informatics, v. 4, n. 4, p. 183-195, 2009. https://doi.org/10.1016/j.ecoinf.2009.07.004

Swain, N. R.; Latu, K.; Christensen, S. D.; Jones, N. L.; Nelson, E. J.; Ames, D. P.; Williams, G. P. A review of open source software solutions for developing water resources web applications. Environmental Modelling \& Software, v. 67, p. 108-117, 2015. https://doi.org/10.1016/j.envsoft.2015.01.014

Tebano, C.; Pasanisi, F.; Grauso, S. QMorphoStream: processing tools in QGIS environment for the quantitative geomorphic analysis of watersheds and river networks. Earth Science Informatics, v. 10, n. 2, p. 257268, 2017. https://doi.org/10.1007/s12145-0160284-0

Yu, H.-L.; Ku, S.-C.; Kolovos, A. A GIS tool for spatiotemporal modeling under a knowledge synthesis framework. Stochastic Environmental Research and Risk Assessment, v. 30, n. 2, p. 665-679, 2016. https://doi.org/10.1007/s00477-015-1078-5

Informação da Licença: Este é um artigo Open Access distribuído sob os termos da Licença Creative Commons Attribution, que permite uso irrestrito, distribuição e reprodução em qualquer meio, desde que a obra original seja devidamente citada. 